\title{
Label-Free Detection of Aflatoxin B1 Using a Nanomechanical Sensor
}

\author{
Xiarong Zhou ${ }^{1, ~ *, ~ S h a n g q u a n ~} \mathrm{Wu}^{1}$, Hong Liu ${ }^{2}$, Xiaoping Wu ${ }^{1}$, Qingchuan Zhang ${ }^{1}$ \\ ${ }^{1}$ Department of Modern Mechanics, University of Science and Technology of China, Hefei, People's Republic of China \\ ${ }^{2}$ Department of Chemical Physics, University of Science and Technology of China, Hefei, People's Republic of China
}

\section{Email address:}

zhouxr@mail.ustc.edu.cn (Xiarong Zhou)

${ }^{*}$ Corresponding author

\section{To cite this article:}

Xiarong Zhou, Shangquan Wu, Hong Liu, Xiaoping Wu, Qingchuan Zhang. Label-Free Detection of Aflatoxin B1 Using a Nanomechanical Sensor. International Journal of Mechanical Engineering and Applications. Vol. 4, No. 2, 2016, pp. 81-87. doi: 10.11648/j.ijmea.20160402.17

Received: February 24, 2016; Accepted: May 7, 2016; Published: May 9, 2016

\begin{abstract}
As a highly sensitive nanomechanical sensor, microcantilever sensor is widely used in biochemical detection. Aflatoxin B1 (AFB1), a hepatocarcinogen widely present in food and food materials, is highly dangerous to human health, and new sensitive methods to detect AFB1 are needed. Here, we developed a microcantilever-array-based immunosensor used in stress mode to detect AFB1 with the obvious advantages of a high sensitivity, rapidity, label-free, quantitative, and ability to be performed in real-time. The microcantilever was functionalized with a sulfhydrylated anti-AFB1 antibody, and an ELISA was used to validate the activity of the antibody on the microcantilever. Deflection of the microcantilever corresponding to different AFB1 concentrations was monitored in real-time. The detection limit of the microcantilever sensor in stress mode was lowered to $0.03 \mathrm{ng} / \mathrm{mL}$ for AFB1, which is a significant improvement in comparison with icELISA or a microcantilever sensor operated in dynamic mode. We also successfully detected AFB1 dissolved in a peanut solution. The microcantilever sensor in stress mode provides a new method for detecting extremely low concentrations of AFB1 and may have great potential for food quality control and public health protection.
\end{abstract}

Keywords: Microcantilever, Nanomechanical Sensor, Aflatoxin B1, Label-Free, Surface Stress

\section{Introduction}

Aflatoxin B1 (AFB1), produced primarily by Aspergillus flavus and Aspergillus parasiticus, is the most potent natural hepatocarcinogen and has been designated as a human liver carcinogen (group I) by the International Agency for Research on Cancer [1-3]. AFB1 has been reported in a variety of food and food materials and mainly enters the food chain through the dietary route in humans and animals $[4,5]$. The intake of AFB1 over a long period of time, even at very low concentrations, may greatly damage human health [6]. The European Commission has set the maximum permissible level of AFB1 in food to $2 \mathrm{ng} / \mathrm{g}$ [7]. Therefore, the development of simple and sensitive techniques to detect low concentrations of AFB1 with quick response times is urgently required.

Many analytical methods are currently available to detect AFB1, including thin layer chromatography [8, 9] and high-performance liquid chromatography [10-12]. Although these techniques are extremely sensitive, they typically require extensive sample pretreatment, tedious procedures and expensive equipment. The enzyme-linked immunosorbent assay (ELISA) is a highly sensitive and selective method for AFB1 detection [13, 14] and is widely used. However, small molecules need to be labeled for this technique, which is often difficult and may affect the sensitivity of the assay.

The microcantilever sensor, a highly sensitive, label-free, real-time, rapid-response, and efficient nanomechanical sensor, may be advantageous [15]. When the microcantilever is operated in stress mode, the stress induced by binding events on its surface causes the microcantilever to bend. The microcantilever, which is functionalized with receptor molecules, can recognize the corresponding analyte, including dangerous pesticides [16, 17], proteins $[18,19]$, heavy metal ions [20,21], drugs [22-24], explosives [25, 26], 
and bacteria [27, 28]. The microcantilever can also be operated in dynamic mode, during which the adsorption or release of a mass alters the resonant frequency of the microcantilever, and this mode is usually used to detect macromolecules [29, 30]. However, the microcantilever in stress mode can be used to detect not only macromolecules $[18,19]$ but also small molecules [22, 23].

Here, we developed a nanomechanical immunosensor to detect AFB1 using the microcantilever array in stress mode. An anti-AFB1 antibody was reacted with a sulfhydrylation reagent to produce a thiol (-SH) group, through which it was immobilized onto the gold surface of the microcantilever. The antibody-functionalized microcantilever was then used to detect different concentrations of AFB1. An ELISA was used to test the activity of the anti-AFB1 antibody that was immobilized on the microcantilever surface.

\section{Materials and Methods}

\subsection{Materials and Apparatus}

The anti-AFB1 monoclonal antibody, 2-iminothiolane hydrochloride, bovine serum albumin (BSA), goat anti-mouse IgG conjugated with horseradish peroxidase (IgG-HRP), aflatoxin G2 (AFG2) and 3,3',5,5'-tetramethylbenzidine (TMB) were purchased from Sigma-Aldrich (St. Louis, MO, USA). AFB1 and an aflatoxin B1-ovalbumin conjugate (AFB1-OVA) were purchased from the Beijing Hapten and Protein Biomedical Institute (Beijing, China). The buffers and solutions used included phosphate-buffered saline (PBS; $0.1 \mathrm{M}$ phosphate buffer containing $0.9 \%$ sodium chloride, $\mathrm{pH} 7.5)$, PBS with $0.1 \%(\mathrm{v} / \mathrm{v})$ Tween 20 (PBST), and stop solution $(2.0 \mathrm{M}$ sulfuric acid).

The 96-well polystyrene microplates were purchased from Costar (Corning, NY, USA). The automated plate-washer (Wellwash 4 MK2) and the microplate reader (Multiskan MK3) were purchased from Thermo (Vantaa, Finland). Silicon microcantilever arrays (Micromotive GmbH, Mainz, Germany) were used. Each array contained eight identical microcantilevers, and the dimensions of each microcantilever were $500 \times 90 \times 1 \mu \mathrm{m} 3$. One side of the microcantilever had a thin film of titanium ( $2 \mathrm{~nm}$ ) covered with a $20 \mathrm{~nm}$ layer of gold. An experimental device received from Concentris $\mathrm{GmbH}$ (Switzerland) was used for microcantilever array immunosensor detection.

\subsection{Microcantilever Array Functionalization}

The sulfhydrylation protocol for the anti-AFB1 antibody was the same as previously described [20, 22]. Briefly, 48.58 $\mu \mathrm{L}$ of a $2.0 \mathrm{mg} / \mathrm{mL} 2$-iminothiolane hydrochloride solution was added to $1.0 \mathrm{~mL}$ of a $10 \mathrm{mg} / \mathrm{mL}$ anti-AFB1 antibody solution. After reacting for $1 \mathrm{~h}$ at room temperature, the mixture was dialyzed against $20 \mathrm{mM}$ PBS buffer (containing $150 \mathrm{mM} \mathrm{NaCl}$ and $1.0 \mathrm{mM}$ EDTA, $\mathrm{pH}$ 7.2) for $48 \mathrm{~h}$. The dialyzed sulfhydrylated anti-AFB1 antibody was stored at $-40^{\circ} \mathrm{C}$.

The procedure to immobilize the sulfhydrylated anti-AFB1 antibody onto the microcantilever array was performed in a microplate well, which was replaced after each step. A new microcantilever array was used in each experiment. Before functionalization, the microcantilever array was immersed in 'piranha dip' $\left[\mathrm{V}\left(\mathrm{H}_{2} \mathrm{O}_{2}\right): \mathrm{V}\left(\mathrm{H}_{2} \mathrm{SO}_{4}\right)=1: 3\right]$ for $4 \mathrm{~min}$, washed four times with deionized water and dried under a gentle stream of nitrogen gas. The array was then immersed in 200 $\mu \mathrm{L}$ of a $50 \mu \mathrm{g} / \mathrm{mL}$ sulfhydrylated anti-AFB1 antibody solution and incubated for $2 \mathrm{~h}$ at $37^{\circ} \mathrm{C}$ for functionalization. The functionalized microcantilever array was washed four times with PBST and was then ready to use.

\subsection{Detection of AFB1 Using Indirect Competitive Enzyme-Linked Immunosorbent Assay (icELISA)}

IcELISA was used as the control method to detect AFB1. A microplate was coated with $100 \mu \mathrm{L}$ of a $1 \mu \mathrm{g} / \mathrm{mL}$ AFB1OVA solution for $3 \mathrm{~h}$. After the microplate was washed four times with PBST, it was blocked with $100 \mu \mathrm{L}$ per well of a BSA $(5 \% \mathrm{w} / \mathrm{w})$ solution for $30 \mathrm{~min}$ and washed four times with PBST. Samples $(100 \mu \mathrm{L})$ of different concentrations of the AFB1 solution were then added to the microplate wells, followed by $100 \mu \mathrm{L}$ of a $150 \mathrm{ng} / \mathrm{mL}$ anti-AFB 1 antibody solution. After being incubated for $30 \mathrm{~min}$, the microplate was washed four times with PBST, and then $100 \mu \mathrm{L}$ of an IgG-HRP solution was added to each well for $30 \mathrm{~min}$. After the wells were washed four times with PBST, $100 \mu \mathrm{L}$ of a TMB substrate solution was added to each well for $20 \mathrm{~min}$. The reaction was stopped by adding $100 \mu \mathrm{L}$ of stop solution per well. The absorbance B of the microplate was read at 450 nm with a microplate reader.

\subsection{Characterization of the Activity of the Antibody Immobilized on the Microcantilever with ELISA}

The activity of the anti-AFB1 antibody immobilized on the microcantilever array was characterized with an ELISA. The microcantilever array was placed in a microplate well, which was replaced after each step. As shown in Figure 3(A), the functionalized microcantilever array (a bare microcantilever array was used as the control) was placed in a microplate well, $200 \mu \mathrm{L}$ of a BSA $(5 \% \mathrm{w} / \mathrm{w})$ solution was added, and the microplate was incubated at $37^{\circ} \mathrm{C}$ for $30 \mathrm{~min}$. After the well was washed four times with PBST, $200 \mu \mathrm{L}$ of an IgG-HRP solution was added, and the microplate was incubated at $37^{\circ} \mathrm{C}$ for $30 \mathrm{~min}$. Any unbound enzyme conjugate on the microcantilever array was removed by washing the well four times with PBST. After the microcantilever array was placed in a new well, $100 \mu \mathrm{L}$ of TMB was added to the well for 30 min, and the reaction was stopped by adding $100 \mu \mathrm{L}$ of stop solution. The microcantilever array was removed, and the absorbance of the remaining solution was read at $450 \mathrm{~nm}$ with a microplate reader.

\subsection{Microcantilever Array Immunosensor Detection}

The functionalized microcantilever array was mounted in a fluid cell $(5 \mu \mathrm{L})$, as shown in Figure 1. PBS solution was injected into the cell with a peristaltic pump. The flow was 
maintained at a constant rate $(0.54 \mathrm{~mL} / \mathrm{h})$ after PBS was injected. All the injected liquids was preheated to $300 \pm 0.01$ $\mathrm{K}$. The temperature of the cell was maintained at $300 \pm 0.01$ $\mathrm{K}$. And the room temperature was maintained at $300 \pm 1 \mathrm{~K}$. The deflection of the microcantilever was measured by monitoring the positions of laser beams reflected from the microcantilever to a position-sensitive detector. After deflection of the microcantilevers in array was equilibrated in running buffer, AFB1 dissolved in PBS was injected into the reaction cell, and deflection of the microcantilever array was monitored.

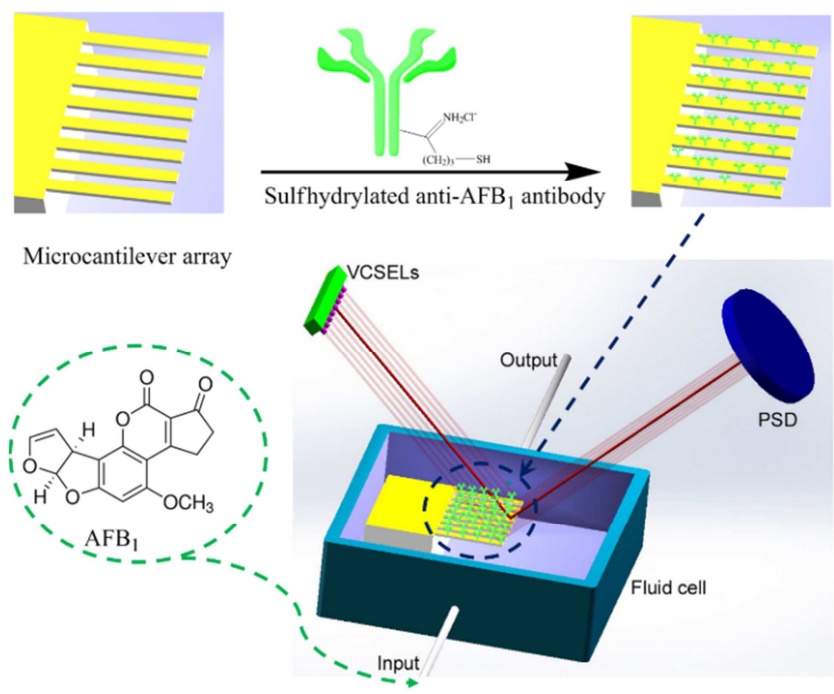

Figure 1. Schematic diagram of the microcantilever array immunosensor and the microcantilever array immobilized with antibody. VCSELs, vertical cavity surface emitting lasers; PSD, position sensitive detector.

\subsection{Preparation of Peanut Samples}

Peanuts (20 g, purchased in a local market) were immersed in $500 \mathrm{~mL}$ of a PBS solution for $24 \mathrm{~h}$, and the peanuts were then removed from the solution. The remaining solution was filtered sequentially through filter papers with pore sizes of $10,5,1.2$, and $0.22 \mu \mathrm{m}$. The filtered solution was called the "peanut solution". Different amounts of standard AFB1 were added to the peanut solution to generate different concentrations of AFB1.

\section{Results and Discussion}

\subsection{Detection of AFB1 with icELISA}

AFB1 (MW = $312 \mathrm{Da})$ is the most potent natural hepatocarcinogen and is recognized as a potential threat to human health, even at low concentrations. The microcantilever array immunosensor was designed to detect low concentrations of AFB1. Before detection, an icELISA was used as a control method to detect AFB1 and measure the binding activity of the anti-AFB1 antibody to AFB1. The measured inhibition curve for AFB1 is shown in Figure 2. $B_{0}$ and $\mathrm{B}$ are the absorbances in the absence and presence of competitor, respectively. The $\mathrm{B} / \mathrm{B}_{0}$ ratio decreased as the AFB1 concentration increased, indicating the inhibitory effect of AFB1 on the binding of AFB1-OVA to the anti-AFB1 antibody. This confirmed the ability of the anti-AFB1 antibody to bind AFB1. The sensitivity of AFB1 detection by icELISA, which was calculated as the concentration that led to $20 \%$ inhibition, was $0.81 \mathrm{ng} / \mathrm{mL}$.

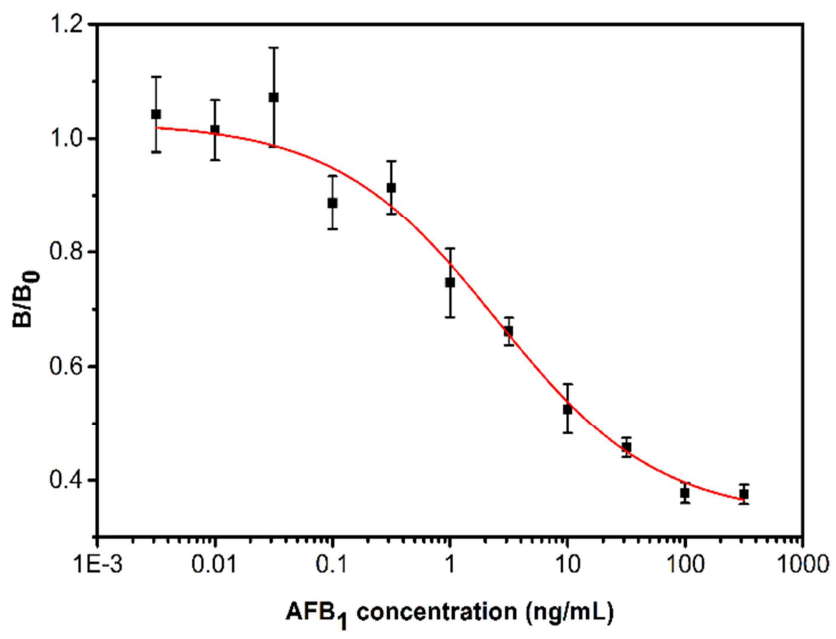

Figure 2. Inhibition curve for AFB1 in an icELISA. The concentration that led to $20 \%$ inhibition by $A F B 1$ was $0.81 \mathrm{ng} / \mathrm{mL}$.

\subsection{Activity of the Antibody on the Microcantilever}

To detect AFB1, the microcantilever must first be modified with a receptor molecule, such as an anti-AFB1 antibody. To confirm that the antibody was successfully immobilized on the microcantilever surface and remained active, an ELISA was used to test its ability to bind to goat anti-mouse IgG (details in Materials and methods). As shown in Figure 3(B), the absorbances $\mathrm{B}$ of the functionalized and naked microcantilevers were 0.235 and 0.052 , respectively, which indirectly verified successful functionalization of the microcantilever array with sulfhydrylated anti-AFB1 antibody and confirmed that the antibody remained active.
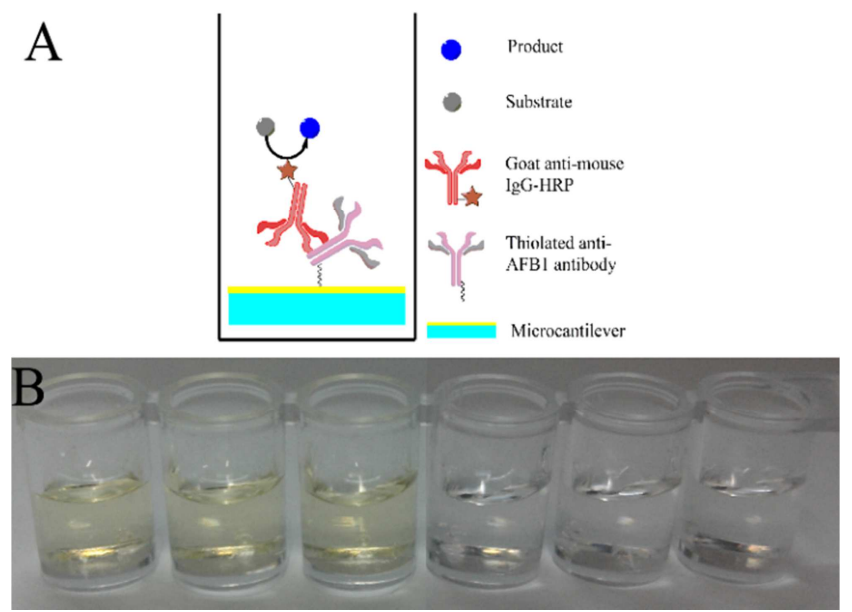

Figure 3. (A) Schematic diagram of the immunological reactions on the microcantilever arrays using ELISA. (B) Characterization of the anti-AFB1 antibody on the microcantilevers. The absorbance $B$ of the anti-AFB1-antibody-functionalized microcantilever $(1, B=0.235 \pm 0.003)$ was compared with that of the naked microcantilever arrays $(2, B=0.052 \pm$ $0.002)$. 


\subsection{Detection of AFB1 by the Microcantilever Array Immunosensor}

Immobilization of the antibody on the microcantilever is important to ensure the sensitivity and stability of the microcantilever immunosensor. Here, the antibody was immobilized onto the gold surface of the microcantilever with the following method. The antibody was sulfhydrylated with the sulfhydrylation reagent 2-iminothiolane hydrochloride, which reacted with the $-\mathrm{NH} 2$ group on the antibody to produce a thiol (-SH) group through which the antibody was conjugated to the gold surface [20,22]. This method required only a single step for antibody immobilization and was highly effective.

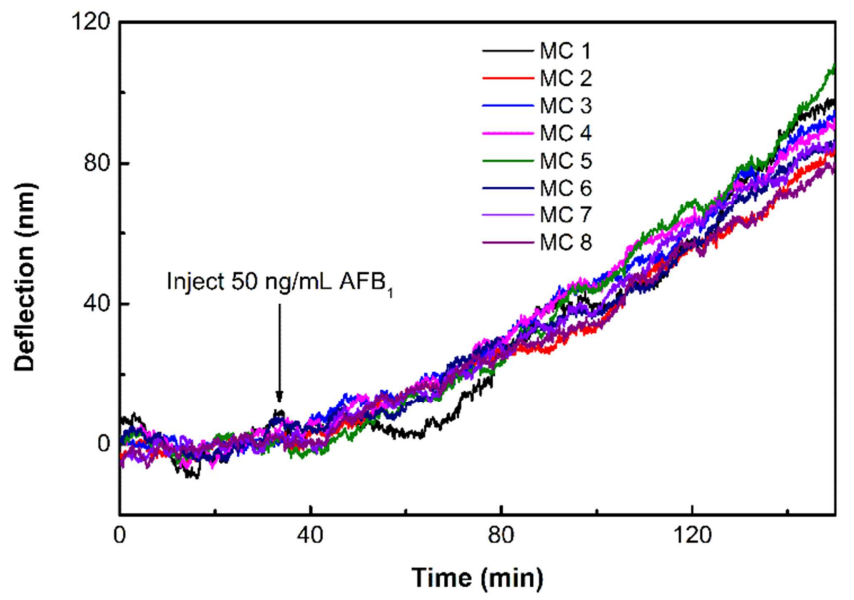

Figure 4. Real-time deflection of each microcantilever in an array in response to $50 \mathrm{ng} / \mathrm{mL} A F B 1 . M C$, microcantilever.

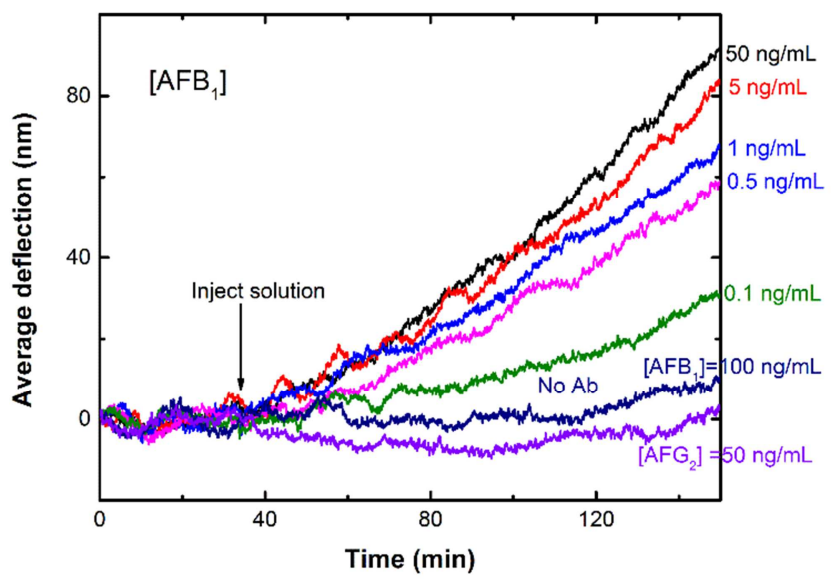

Figure 5. Average microcantilever deflections as a function of time at various concentrations of AFB1. The microcantilever arrays were functionalized with a sulfhydrylated anti-AFB1 antibody. The average deflection represents the mean deflection of the identically functionalized microcantilevers within an array.

For a microcantilever array sensor, the deflection of identically treated microcantilevers must be the same. To examine the response consistency of the different microcantilevers in an array, we measured the deflection curves of all of the microcantilevers within an array in response to $50 \mathrm{ng} / \mathrm{mL} \mathrm{AFB} 1$. All of the microcantilevers in the array were functionalized with the AFB1 antibody. After baseline deflection of the microcantilever array was stabilized in PBS for $33 \mathrm{~min}, 50 \mathrm{ng} / \mathrm{mL}$ AFB1 solution dissolved in PBS was injected, as shown in Figure 4. Deflections of the different microcantilevers in the array were very similar $(91.5 \pm 9.1 \mathrm{~nm})$, indicating that the microcantilever array immunosensor had good consistency for detecting AFB1.

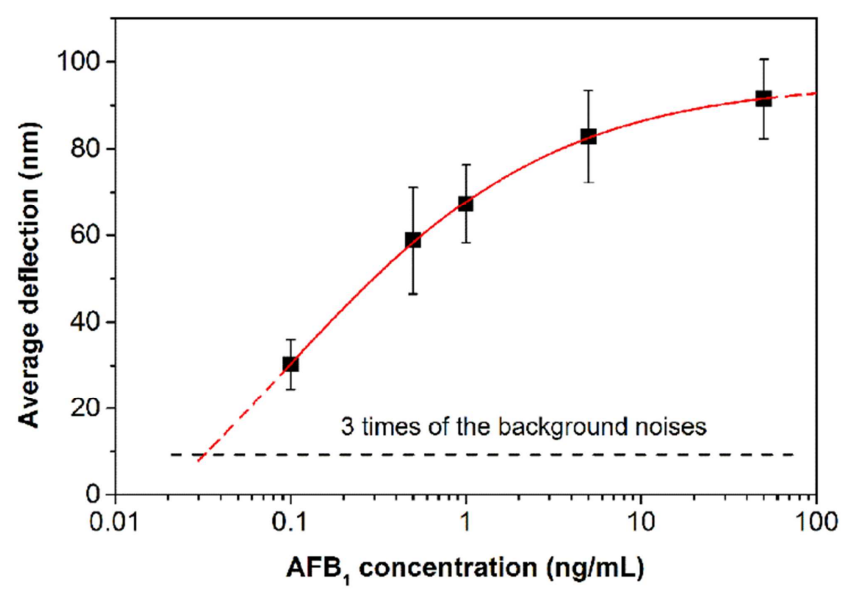

Figure 6. Maximum deflection of the microcantilever array at various concentrations of $A F B 1$.

To investigate the relationship between microcantilever deflection and AFB1 concentration, we measured microcantilever deflections induced by different concentrations of AFB1. After the baseline of microcantilever deflection was stabilized in PBS solution, different concentrations of AFB1 dissolved in PBS were circulated through the fluid cell, respectively. The deflection curves of the microcantilevers in response to various AFB1 concentrations are shown in Figure 5. The average deflection represents the mean bending value of identically functionalized microcantilevers within an array. We defined microcantilever bending toward the gold layer as positive (up). The binding between the anti-AFB1 antibody and AFB1 caused the microcantilever to bend toward the gold surface so that tensile stress occurred on the gold surface. The average bending signals at $150 \mathrm{~min}$ for AFB1 solutions of 50, $5,1,0.5$, and $0.1 \mathrm{ng} / \mathrm{mL}$ were $92,83,67,59$, and $30 \mathrm{~nm}$, respectively. When the reference microcantilever without antibody was exposed to $100 \mathrm{ng} / \mathrm{mL}$ AFB1, the microcantilever did not show a significant response. Moreover, a significant response was also not observed when $50 \mathrm{ng} / \mathrm{mL}$ AFG2, which is a toxin in food and food products but does not react with anti-AFB1 antibody, was added to the cell. These results indicated that the bending of the microcantilever array was caused by the specific binding interaction between the anti-AFB1 antibody and AFB1.

The maximum microcantilever deflection caused by the specific binding of the anti-AFB1 antibody to AFB1 in the test period is shown in Figure 6. Each data point and error bar represents the mean deflection value and standard deviation, respectively, for identically functionalized 
microcantilevers within an array. The responses of the microcantilever array showed a positive correlation between the deflection and the concentration of AFB1, indicating that the microcantilever can quantitatively detect the analyte. The background noise of the measurement was approximately 3 $\mathrm{nm}$, so the limit of detection (LOD) of the microcantilever sensor, which was calculated as three times higher than the background noise, was approximatively as low as 0.03 $\mathrm{ng} / \mathrm{mL}$ AFB1. Our results demonstrated that the microcantilever operated in stress mode was sensitive and suitable to detect AFB1.

The surface stress generated by the binding interaction between a small molecule and its antibody was a tensile stress in the present study, which differs from the compressive stress induced by the binding interaction between a macromolecule and its antibody [17, 31, 32]. Our findings are consistent with the results of another study [17]. The stress on the microcantilever surface may have originated from the conformational changes induced in the antibody when it bound to the antigen, manifesting as the motion of the Fab arm, which exposed the C1q-binding site on the antibody, as proposed by $\mathrm{Wu}$ et al. [17]. This generated a stress difference between the AFB1-antibody-modified surface on the upper side of the microcantilever and the silicon surface on the lower side.

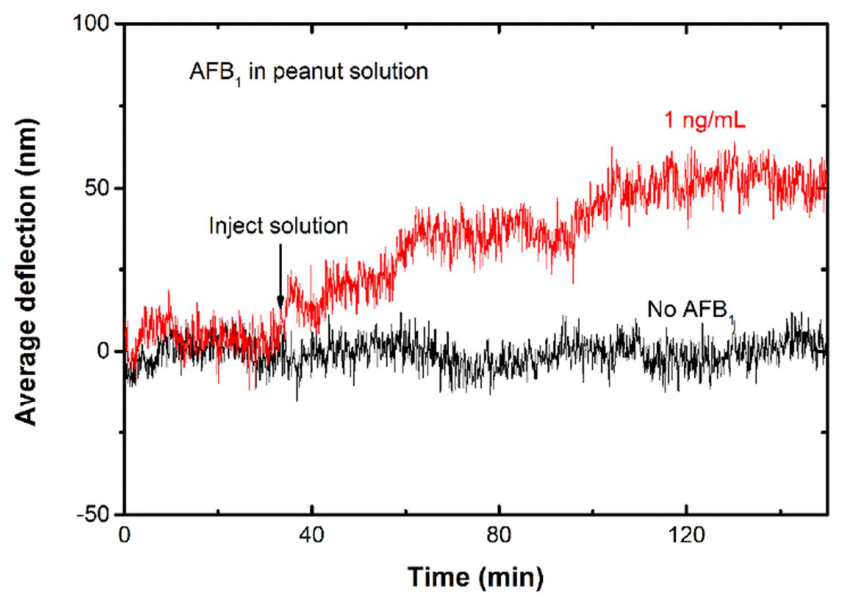

Figure 7. Real-time average deflection of the microcantilever array for AFB1 dissolved in a peanut solution.

A microcantilever sensor operated in dynamic mode was also used to detect total aflatoxins with an LOD of $3 \mathrm{ng} / \mathrm{mL}$, as reported by Ricciardi et al. [33]. The LOD of icELISA for AFB1 was $0.81 \mathrm{ng} / \mathrm{mL}$ (Section 3.1). Therefore, compared with other methods, the microcantilever immunosensor in stress mode that we developed here showed a significant improvement in sensitivity. The large sensitivity difference between the microcantilever in stress and dynamic modes is interesting to investigate. The frequency shifts of microcantilevers in dynamic mode are mainly caused by added mass, and this mode is usually used to detect macromolecules [29, 30, 34]. However, the weight of a molecule, such as AFB1 $(\mathrm{MW}=312 \mathrm{Da})$ is so small that the added mass to the microcantilever was low, and thus the frequency shift of the microcantilever induced by adsorption of a small amount of AFB1 may not have been large enough. Therefore, the microcantilever in dynamic mode showed a lower sensitivity for AFB1. The sensitivity of the microcantilever for AFB1 in the present study demonstrated a 100-fold improvement in comparison with the microcantilever in dynamic mode. The deflection of the microcantilever in stress mode was not determined by the adsorbed mass but may be attributed to the conformational changes in the anti-AFB1 antibody that were induced when it bound to AFB1, which may have caused a large energy release from the anti-AFB1 antibody, similarly to that released from a compressed spring. Therefore, trace amounts of AFB1 can cause sufficient deflection of the microcantilever, and thus the microcantilever in stress mode showed higher sensitivity.

\subsection{Detection of AFB1 in Peanuts}

AFB1 that is present in peanuts has become a threat to human health. Therefore, we used the microcantilever array immunosensor to detect AFB1 dissolved in a peanut solution. The average deflection profile of the microcantilever for AFB1 in a peanut solution is shown in Figure 7. The average deflection of the microcantilevers at $150 \mathrm{~min}$ was approximately $51 \mathrm{~nm}$ for $1 \mathrm{ng} / \mathrm{mL}$ AFB1 in the peanut solution, which was close to the deflection (approximately 67 $\mathrm{nm}$ ) for $1 \mathrm{ng} / \mathrm{mL}$ AFB1 in PBS. No significant response was observed when the peanut solution without AFB1, which was used as a reference, was injected into the cell. The fluctuations in the mean deflection induced by the injection of AFB1 dissolved in the peanut solution (Figure 7) were greater than the fluctuations in the deflection induced by the injection of AFB1 dissolved in PBS (Figure 5), which may indicate that the simply treated peanut solution contained more complex substances than the PBS solution. These data show that the microcantilever immunosensor can detect AFB1 in peanut solutions that contain complex components, indicating that the microcantilever can be used for on-site AFB1 detection.

\section{Conclusion}

In conclusion, we showed that the microcantilever array operated in stress mode can sensitively detect AFB1 without the need for a label in both PBS and peanut solutions. A sulfhydrylated anti-AFB1 antibody was immobilized onto the gold surface of the microcantilever and an ELISA was used to validate the activity of the antibody on the microcantilever. The deflection increased with increasing concentrations of AFB1, indicating that the microcantilever sensor can quantitatively detect AFB1. The LOD of the microcantilever operated in stress mode for AFB1 was as low as $0.03 \mathrm{ng} / \mathrm{mL}$, which is a significant improvement in comparison with icELISA $(0.81 \mathrm{ng} / \mathrm{mL})$ or a microcantilever operated in dynamic mode $(3 \mathrm{ng} / \mathrm{mL})$. The microcantilever sensor was successfully used to detect AFB1 in a peanut solution, showing its capacity to detect the analyte on site in a 
complex environment. The results indicate that the microcantilever in stress mode is well suited to detect small molecules with significantly low LOD. Passivation of $\mathrm{Si}$ surface, on-chip reference and antibody immobilization, however, may affect the detection sensitivity and reliability. Thus, further research should focus on these.

\section{Acknowledgements}

This work was supported by the National Natural Science Foundation of China (Grant No. 11502265, 11127201), Chinese National Key Technology R\&D Program (2012BAK08B05), and Fundamental Research Funds for the Central Universities (WK2090050032).

\section{References}

[1] International Agency for Research on Cancer, IARC monographs on the evaluations of carcinogenic risks to humans, IARC, Lyon, 1993, vol. 56, pp. 245-395.

[2] R. Chauhan, P. R. Solanki b, J. Singh, I. Mukherjee, T. Basu, B. D. Malhotra, A novel electrochemical piezoelectric label free immunosensor for aflatoxin B1 detection in groundnut, Food Control 52 (2015) 60-70.

[3] A. T. Mata a, J. P. Ferreira, B. R. Oliveira, M. C. Batoréu, M. T. Barreto Crespo, V. J. Pereira, M.R. Bronze, Bottled water: Analysis of mycotoxins by LC-MS/MS, Food Chem. 176 (2015) 455-464.

[4] I. Y. S. Rustom, Aflatoxin in food \& feed: occurrence, legislation \& inactivation by physical methods, Food Chem. 59 (1997) 57-67.

[5] E. Papp, K. H-Otta, G. Záray, E. Mincsovics, Liquid chromatographic determination of aflatoxins, Microchem. J. 73(1-2) (2002) 39-46.

[6] M. Miraglia, C. Brera, M. Colatosti, Application of biomarkers to assessment of risk to human health from exposure to mycotoxins, Microchem. J. 54 (1996) 472-477.

[7] Commission Recommendation (EC) no1881/2006 of 19 December 2006 setting maximum levels for certain contaminants in foods tuffs, Official Journal of the European Union 364, 2006, pp. 5-24.

[8] J. Stroka, R. van Otterdijk, E. Anklam, Immunoaffinity column clean-up prior to thin-layer chromatography for the determination of aflatoxins in various food matrices, J. Chromatogr. A 904 (2000) 251-256.

[9] A. Fernandez, R. Belio, J. J. Ramos, M. C. Sanz, T. Saez, Aflatoxins and their metabolites in the tissues, faeces and urine from lambs feeding on an aflatoxin-contaminated diet, J. Sci. Food Agr. 74 (1997) 161-168.

[10] J. Jaimez, C. A. Fente, B. I. Vazquez, C. M. Franco, A. Cepeda, G. Mahuzier, P. Prognon, Application of the assay of aflatoxins by liquid chromatography with fluorescence detection in food analysis, J. Chromatog. A 882 (2000) 1-10.

[11] E. Beltrán, M. Ibáñez, J. V. Sancho, M. Á. Cortés, V. Yusà, F. Hernández, UHPLC-MS/MS highly sensitive determination of aflatoxins, the aflatoxin metabolite M1 and ochratoxin A in baby food and milk, Food Chem. 126 (2011) 737-744.

[12] A. L. Capriotti, C. Cavaliere, P. Foglia, R. Samperi, S. Stampachiacchiere, S. Ventura, A. Lagana, Multiclass analysis of mycotoxins in biscuits by high performance liquid chromatography-tandem mass spectrometry. Comparison of different extraction procedures, J. Chromatogr. A 1343 (2014) 69-78.

[13] P. C. Turner, K. H. Dingley, J. Coxhead, S. Russell, C. R. Garner, Detectable levels of serum aflatoxin B1-albumin adducts in the United Kingdom population: implications for aflatoxin-B1 exposure in the United Kingdom, Cancer Epidemiol. Biomar. 7 (1998) 441-447.

[14] B. H. Liu, Y. T. Hsu, C. C. Lu, F. Y. Yu, Detecting aflatoxin B1 in foods and feeds by using sensitive rapid enzyme-linked immunosorbent assay and gold nanoparticle immunochromatographic strip, Food Control 30 (2013) 184-189.

[15] K. R. Buchapudi, X. Huang, X. Yang, H. Ji, T. Thundat, Microcantilever biosensors for chemicals and bioorganisms, Analyst 136 (2011) 1539-1556.

[16] C. R. Suri, J. Kaur, S. Gandhi, G. S. Shekhawat, Label-free ultra-sensitive detection of atrazine based on nanomechanics, Nanotechnology 19 (2008) 5502-5507.

[17] S. Wu, T. Nan, C. Xue, T. Cheng, H. Liu, B. Wang, Q. Zhang, X $\mathrm{Wu}$, Mechanism and enhancement of the surface stress caused by a small-molecule antigen and antibody binding, Biosens. Bioelectron. 48 (2013) 67-74.

[18] G. Wu, R. H. Datar, K. M. Hansen, T. Thundat, R. J. Cote, A. Majumdar, Bioassay of prostate-specific antigen (PSA) using microcantilevers, Nat. Biotechnol. 19 (2001) 856-860.

[19] K. Gruber, T. Horlacher, R. Castelli, A. Mader, P. H. Seeberger, B. A. Hermann, Cantilever array sensors detect specific carbohydrate-protein interactions with picomolar sensitivity, ACS Nano 5 (2011) 3670-3678.

[20] H. Zhao, C. Xue, T. Nan, G. Tan, Z. Li, Q. X. Li, Q. Zhang, B. Wang, Detection of copper ions using microcantilever immunosensors and enzyme-linked immunosorbent assay, Anal. Chim. Acta 676 (2010) 81-86.

[21] X. Chen, W. Chen, U. Mohideen, Application of displacement principle for detecting heavy metal ions and EDTA using microcantilevers, Sensor. Actuat. B 161 (2012) 203-208.

[22] C. Xue, H. Zhao, H. Liu, Y. Chen, B. Wang, Q. Zhang, X. Wu, Development of sulfhydrylated antibody functionalized microcantilever immunosensor for taxol, Sensor. Actuat. B 156 (2011) 863-866.

[23] X. Bai, H. Hou, B. Zhang, J. Tang, Label-free detection of kanamycin using aptamer-based cantilever array sensor, Biosens. Bioelectron. 56 (2014) 112-116.

[24] L. Huang, Y. Pheanpanitporn, Y. Yen, et al, Detection of the antiepileptic drug phenytoin using a single free-standing piezoresistive microcantilever for therapeutic drug monitoring, Biosens. Bioelectron. 59 (2014) 233-238.

[25] D. Lee, S. Kim, S. Jeon, T. Thundat, Direct detection and speciation of trace explosives using a nanoporous multifunctional microcantilever, Anal. Chem. 86 (2014) 5077-5082. 
[26] F. G. Bosco, M. Bache, E.-T. Hwu, C. H. Chen, S. S. Andersen, K. A. Nielsen, S. S. Keller, J. O. Jeppesen, I.-S. Hwang, A. Boisen, Statistical analysis of DNT detection using chemically functionalized microcantilever arrays, Sensor. Actuat. B 171-172 (2012) 1054-1059.

[27] A. Mader, K. Gruber, R. Castelli, B. A. Hermann, P. H. Seeberger, J. O. Rädler, M. Leisner, Discrimination of Escherichia coli strains using glycan microcantilever array sensors, Nano Lett. 12 (2012) 420-423

[28] K. Nieradka, K. Kapczynska, J. Rybka, T. Lipinski, P. Grabiec, M. Skowickid T. Gotszalka, Microcantilever array biosensors for detection and recognition ofGram-negative bacterial endotoxins, Sensor. Actuat. B 198 (2014) 114-124.

[29] P. M. Kosaka, V. Pini, J. J. Ruz, R. A. da Silva, M. U. González, D. Ramos, M. Calleja, J. Tamayo1, Detection of cancer biomarkers in serum using a hybrid mechanical and optoplasmonic nanosensor. Nat. Nanotechnol. 9 (2014), 1047-1053.

[30] T. Braun, M. K. Ghatkesar, N. Backmann, W. Grange, P. Boulanger, L. Letellier, H. Lang, A. Bietsch, C. Gerber, M.
Hegner, Quantitative time-resolved measurement of membrane protein-ligand interactions using microcantilever array sensors, Nat. Nanotechnol. 4 (2009) 179-185.

[31] V. Dauksaite, M. Lorentzen, F. Besenbacher, J. Kjems, Antibody-based protein detection using piezoresistive cantilever arrays, Nanotechnology, 18 (2007) 125503-125508.

[32] Y. Arntz, J. D. Seelig, H. P. Lang, G. S. Shekhawat, Label-free protein assay based on a nanomechanical cantilever array, Nanotechnology, 14 (2003) 86-90.

[33] C. Ricciardi, R. Castagna, I. Ferrante, F. Frascella, S. L. Marasso, A. Ricci, G. Canavese, A. Lore, A. Prelle, M. L. Gullino, D. Spadaro, Development of a microcantilever-based immunosensing method for mycotoxin detection, Biosens. Bioelectron. 40 (2013) 233-239.

[34] J. Tamayo, P. M. Kosaka, J. J. Ruz, A. S. Paulo, M. Calleja, Biosensors based on nanomechanical systems, Chem. Soc. Rev. 42 (2013) 1287-1311. 